\title{
Integrated approach for accurate localization of optic disc and macula
}

\author{
Vaanathi Sundaresan ${ }^{1}$, Keerthi Ram², Niranjan Joshi ${ }^{2}$, Mohanasankar \\ Sivaprakasam $^{1,2}$ and Rashmin Gandhi ${ }^{3}$ \\ 1 Indian Institute of Technology Madras (IITM), India \\ 2 Healthcare Technology Innovation Centre, IITM, India \\ 3 Centre for Sight, India
}

\begin{abstract}
The location of three main anatomical structures in the retina namely the optic disc, the vascular arch, and the macula is significant for the analysis of retinal images. Presented here is a novel method that uses an integrated approach to automatically localize the optic disc and the macula with very high accuracy even in the presence of confounders such as lens artifacts, glare, bright pathologies and acquisition variations such as non-uniform illumination, blur and poor contrast. Evaluated on a collective set of 579 diverse pathological images from various publicly available datasets, our method achieves sensitivity $>99 \%$ and normalized localization error $<5 \%$ for optic disc and macula localization.
\end{abstract}

\section{Introduction}

Optic disc (OD), also known as optic nerve head (beginning of optic nerve) is a roughly circular bright structure in the retinal fundus from which the retinal blood vessel tree emerges. Macula, on the other hand, is a dark avascular region in the retina responsible for central vision. Both these structures are vital parts of the retina, and establish visual landmarks for describing other anatomical and pathological structures. Detection of OD is required for identifying and quantifying glaucoma. Any pathology near the macular center directly affects the central vision, signifying advanced stages of retinal diseases like age-related macular degeneration, maculopathy and diabetic retinopathy (DR). Hence, detection of OD and macula is an essential step in disease screening and grading disease severity.
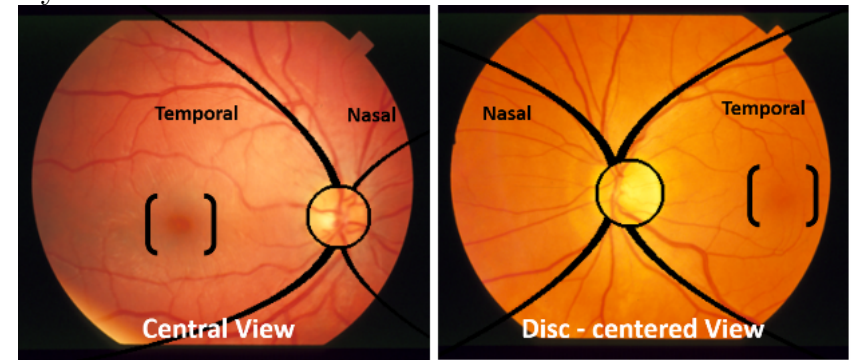

Fig. 1. Two retinal views (central view, disc-centered view) showing OD, macula, arcades on nasal and temporal side 


\section{$1.1 \quad$ Prior work}

For the detection of OD, existing methods exploit specific characteristics such as shape, intensity, e.g., Hough circle transform [1], bright region segmentation, matched filter [2] and feature based classification [3]. Though such methods perform satisfactorily in majority of cases, they might lead to false detection in the case of poor contrast, faint or indistinct OD, presence of pathologies including Geographic Atrophy (GA) and very large exudates.

An alternative is to use indirect attributes, relying on domain information: emergence of retinal blood vessels from the OD. In [4] fuzzy convergence of vessels is used to detect OD. In [5] vessel directions were found by utilizing the information that the convergence of vessels occur at OD position. Both these approaches have indicated high accuracy in a challenging dataset [4]. A reported limitation, however, is the dependence on sufficiently reliable blood vessel segmentation.

Some of the prominent work for the detection of macula are low intensity pixel clustering, template matching [6] and utilization of parabolic model [7] along with retinal raphe [8]. Performance of intensity based methods for macula detection are usually hindered by pathology such as GA. The macula by itself is not a conspicuous structure, and darker images (due to pigmentation, low illumination) might pose a challenge for reliable detection.

To address the drawbacks of individual methods used in existing work, we propose an approach which converts the inter-dependency of the three anatomical structures: OD, macula and vessels, into an iterative inter-refinement process. Our proposed approach consists of an unconventional method of utilizing blood vessels for refining the symmetry of vessel map, estimating the distance transform and determining an estimate for macula location. The highlight of our approach is the integration of multiple strategies including intensity information, characterization of symmetric vessel structure and the anatomical cues such as distance of macula from OD, intensity and avascularity of macula. By combining the strengths of the individual strategies, our approach renders a reliable localization overcoming imaging artifacts, acquisition variation and pathologies.

\section{Proposed method}

Our proposed method is targeted at two widely used views of the retina captured in retinal images (refer fig. 1). Our proposed method consists of three important steps: 1 . Refining the symmetry of arcade by iteratively redetermining axis of symmetry, 2. Modeling the retinal arcades and estimating the individual estimates for OD and macula centers, 3. Integration of multiple approaches to arrive at final estimate of OD and macula center. Given an image of unspecified view, our method progresses as follows (refer fig. 3):

We extract the crude form of the vascular arcade $V_{0}$ (refer fig. 2) by adopting a simple vessel detection step based on morphology (supremum of opening with rotated linear structuring elements). 

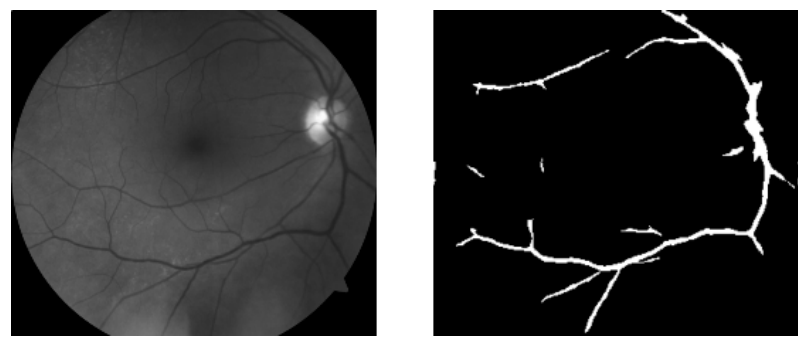

Fig. 2. Computation of crude arcade $V_{0}$ from grayscale channel

From $V_{0}$, we iteratively estimate the axis of symmetry $X_{i}$, and refine $V_{i}$ so that the arcade pixels are progressively selected to be symmetric about the axis

$$
\begin{gathered}
X_{i}=\text { estimate_symmetry }\left(V_{i}\right) \\
V_{i+1}=\text { refine_arcade }\left(V_{i}, X_{i}\right)
\end{gathered}
$$

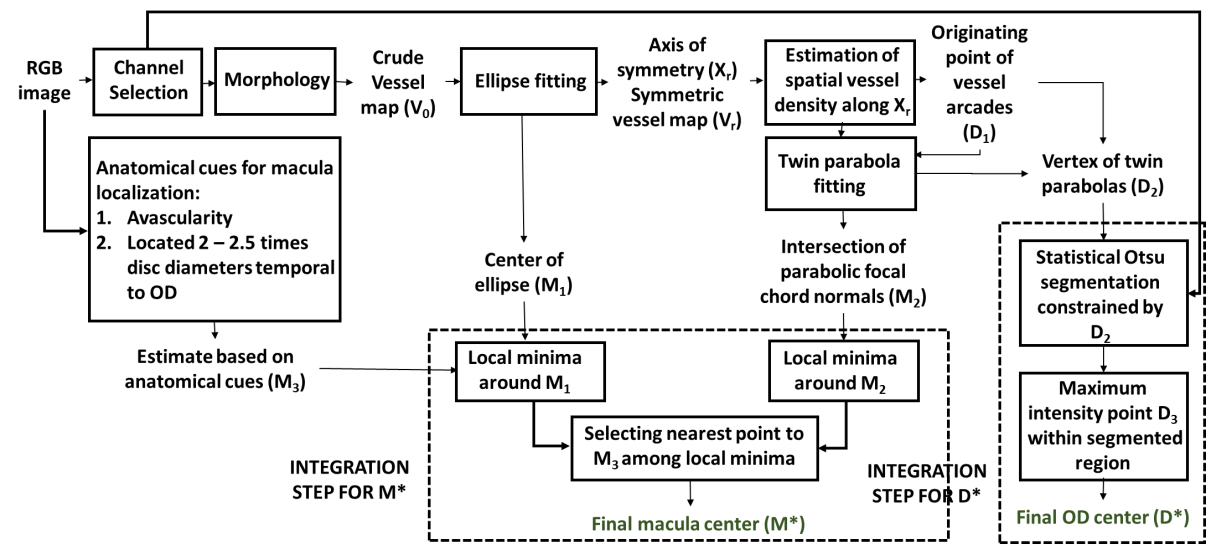

Fig. 3. Flowchart depicting the process of localization of OD and macula

The three individual estimates of OD are as follows (refer fig. 4):

Estimation of initial OD location: Using the refined axis of symmetry $X_{r}$, the initial guess of OD location $D_{1}$ is made by examining a band of pixels around the axis, to spot convergence of blood vessel pixels.

Parabolic model fitting on arcade: The arcades of the refined vessel structure $V_{r}$ are modeled by fitting twin parabolas and their common vertex $D_{2}$ is the second estimate of OD location (though their axes are independent). $D_{1}$ and $X_{r}$ provide initialization for vertex and axes of the parabolas respectively.

Intensity based OD localization: The third estimate $D_{3}$ of OD location is the maximum intensity point within the segmented region obtained by a statistical thresholding method constrained by a window around $D_{2}$.

We thus impose transitive dependency between the above determined estimates of OD position, whereas the estimates of macula are independent, aiding in precise macula localization irrespective of magnitude of error in OD localization. The macula estimates are (refer fig. 4 and 5):

From major arcade fitting: The intersection of focal chord normals of the fitted major arcade parabola provides an estimate of macula position $M_{2}$. 

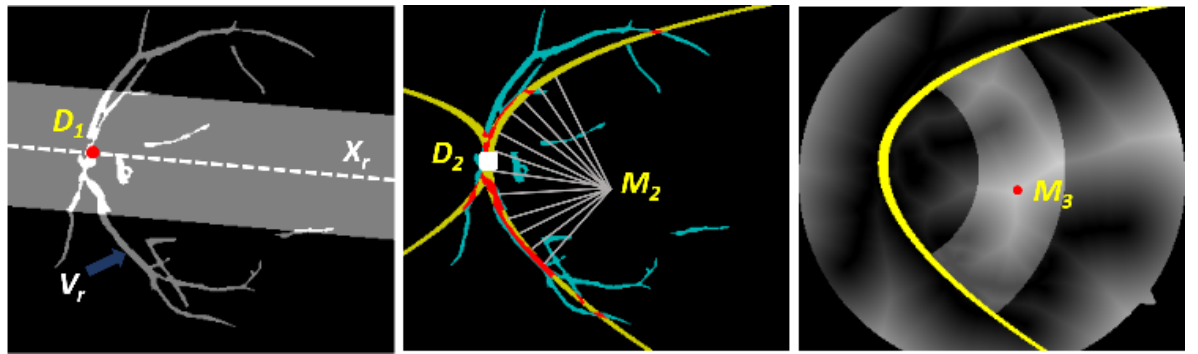

Fig. 4. (1) Computation of $D_{1}$ from $V_{r}$ and $X_{r}$, (2) Twin-parabola fit and $D_{2}$ localization, focal chord intersection of major arcade parabola $\left(P^{+}\right)$to define $M_{2},(3)$ Localization of $M_{3}$ using distance transform from anatomical cues

From anatomy: The macula is at a distance of 2-2.5 'disc diameters' temporal to the optic disc [8], flanked by the major arcade, and is avascular. These characteristics are applied to compute macula estimate $M_{3}$. We compute a binary distance transform of $V_{r}$, and locate $M_{3}$ as the maximum (furthest from vessels) within an annulus centered on $D_{2}$ and radius set using the focal length of the major parabola.

From axis determination step: An estimate of the macula position $M_{1}$ comes directly from our method of determining axis of symmetry. This is elaborated in the following section.

\subsection{Determining axis of symmetry}

The crux of our approach is a reliable identification of symmetry of the vasculature from crude arcade $V_{0}$. We use an iterative model-fitting approach, where our chosen parametric curve models the vessels diverging at the OD and converging around the macula. Our model of choice is ellipse due to its axial symmetry, and the method of fit we use is a nonlinear least-squares algorithm with pragmatic bounds on the major and minor axis lengths. For least-squares fit, we use 'Trust Region Reflective' optimization algorithm, since it handles bound constraints better than LM optimization. The major axis of the fitted ellipse gives the axis of symmetry $X$ of the major arcade.

Due to image variations (illumination, contrast, imaging artifacts) and pathology, the data $\left(V_{0}\right)$ supplied to the fitting routine might not be sufficiently reliable for producing a structurally descriptive elliptic model of the major arcade. We treat this as a problem of model fitting in the presence of partial data and noisy data. Our solution is two-fold: 1 . Refining the apparent symmetry in the input, and recomputing the model fit by iteratively reselecting the input data $V_{i}$, 2. Robust fit using RANSAC

At the end of each iteration, the axis $X_{i}$ is used to divide $V_{i}$ into two, and in each half, a top-percentile threshold and skeletonization are applied to compute $V_{i+1}$. Iteration terminates when there is no change in $V_{i+1}$. Three outcomes arise from this method: $1 . X_{r}$ the refined axis of symmetry at convergence, represents an estimate of the retinal raphe, along which the OD and macula are expected to lie, 2. $V_{r}$ a refined arcade, which is suitable for fitting twin parabolas (discussed next), 3. $M_{1}$ the center of the ellipse, which is an estimate of the macula position. 


\subsection{Modeling the retinal arcades}

In order to generalize across the two views (central view, disc-centered view) we fit twin parabolas on $V_{r}$, providing initialization from $X_{r}$ and $D_{1}$ (fig. 4). One of the parabolas $\left(P^{+}\right)$represent the major temporal arcade which encompasses the brighter side of OD, while its counterpart $\left(P^{-}\right)$represents the nasal arcade. From the fit, the parabola with greater number of inlying vessel pixels is named as $P^{+}$, since the major arcade arterial and venous vessels occupy more pixels compared to nasal arcade. If the number of inliers for $P^{+}$and $P^{-}$are comparable, it signifies that the image might be of disc-centred view. In such case, we examine the intensity enclosed by the two parabolas near the vertex, and consider the brighter of the two as $P^{+}$. Also in such case, we regard the estimate of macula $M_{1}$ as unreliable.

\subsection{Integrating multiple approaches}

We integrate these position estimates for OD and macula, to arrive at the location that best matches with the anatomical position, by building on their individual strengths.
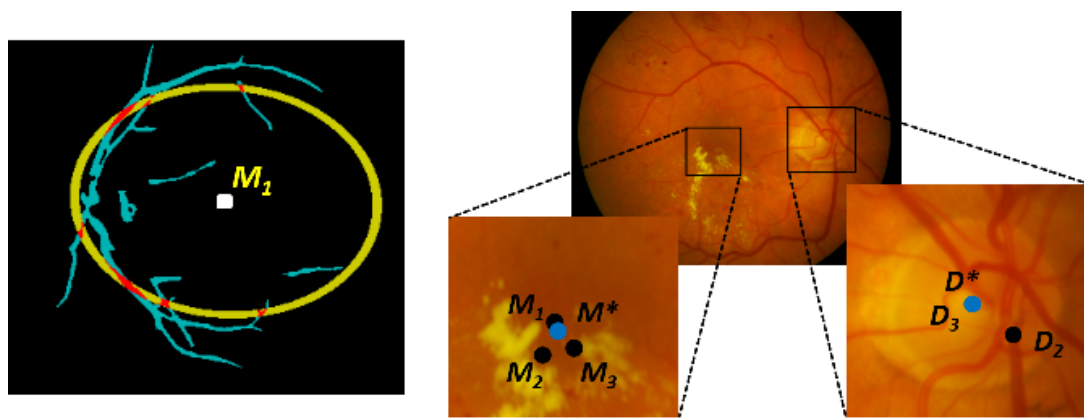

Fig. 5. (1) $M_{1}$ from the symmetry model, (2) Integration of position estimates obtained from multiple approaches

The estimate from curve fitting method $D_{2}$ localizes the OD region accurately while $D_{3}$ gives precise estimation of OD center. By spatially confining our search space around $D_{2}$, we avoid other bright pathologies and confounders. Hence, we assign $D_{3}$ as final estimate $D^{*}$ of OD location (fig. 5). Further, as a preprocessing step, we dynamically select the channel of best contrast for the particular image, and apply a non-linear (gamma) stretch in the brighter intensities (above background level). The main false positives in the estimation of $D_{3}$ such as RNFL and choroidal blood vessels are overcome by channel selection and gamma stretch.

Depending on type of view, the reliability of $M_{1}$ and $M_{2}$ changes. Thus, the estimate $M_{3}$, which is obtained from binary distance transform of $V_{r}$ using anatomical information, is used here in addition to vessel attributes. The estimates $M_{1}$ and $M_{2}$ are shifted to their local minima within a specified window, since they might not naturally coincide with intensity minima. By spatially confining our search space by $M_{3}$, we determine the final estimate for macula center $M^{*}$ by choosing the point among local minima nearer to $M_{3}$ (fig. 5 ). 


\section{Results and discussion}

We have evaluated the performance of our method on publicly available datasets: DIARETDB1 (89 images), DMED (169 images), MESSIDOR (100 images), DRIVE (40 images), STARE (81 images used in [4] and [5]), and a dataset of 100 pathological images collected from a local hospital. The cumulative collection forms a representative dataset (Combined) consisting of 579 images, of which 416 images $(71.8 \%)$ are pathological. The collection consists of mydriatic and non-mydriatic images with diverse pathologies and varying angles of acquisition. STARE and local datasets include disc-centered view images too. STARE has challenging images with imaging artifacts, and severe pathology, with OD either partially or completely in view.

We apply a rigorous evaluation metric for the localization of OD and macula, based on which we determine sensitivity in a stringent manner. Previously, [9] used this measure for OD localization, by normalizing the mean error. We express the accuracy of localization in terms of normalized error for each image:

$$
\xi_{\text {disc }}=\frac{\left\|D_{g t}-D^{*}\right\|}{\text { disc_dia }} \quad \xi_{m a c}=\frac{\left\|M_{g t}-M^{*}\right\|}{\text { disc_dia }}
$$

expressed as a percentage error value, with respect to expert annotation of disc center $D_{g t}$, macula center $M_{g t}$ and disc diameter disc_dia. We consider a detection as true-positive if $\xi \leq 40 \%$.

Table 1. Mean percentage error among true-positives, sensitivity in different datasets, in decreasing order of $\xi_{\text {disc }}\left(D^{*}\right)$. Number of false-positives: OD - 5, Macula - 2

\begin{tabular}{|c|c|c|c|c|c|c|c|}
\hline \multirow{3}{*}{$\begin{array}{l}\text { Dataset } \\
\text { (No of images) } \\
\end{array}$} & \multirow{2}{*}{\multicolumn{2}{|c|}{$\begin{array}{c}D \\
\text { localization } \\
\xi_{d i s c}\end{array}$}} & \multirow{2}{*}{\multicolumn{3}{|c|}{$\begin{array}{c}\text { Macula } \\
\text { localization } \\
\xi_{m a c}\end{array}$}} & \multirow{2}{*}{\multicolumn{2}{|c|}{$\begin{array}{l}\text { Detection } \\
\text { sensitivity }\end{array}$}} \\
\hline & & & & & & & \\
\hline & $\begin{array}{l}D_{2} \\
(\%)\end{array}$ & $\begin{array}{l}D^{*} \\
(\%)\end{array}$ & $\begin{array}{l}M_{1} \\
(\%)\end{array}$ & $\begin{array}{l}M_{2} \\
(\%)\end{array}$ & $\begin{array}{l}M^{*} \\
(\%)\end{array}$ & $\begin{array}{l}O D \\
(\%)\end{array}$ & $\begin{array}{c}\text { Macula } \\
(\%)\end{array}$ \\
\hline STARE (20) & 11.45 & 9.79 & 15.99 & 13.05 & 11.93 & 95 & 100 \\
\hline${ }^{\mathrm{DC}}$ Local (37) & 2.93 & 1.20 & 7.13 & 5.66 & 5.59 & 100 & 100 \\
\hline STARE (61) & 15.66 & 9.63 & 15.64 & 20.5 & 11.13 & 95.08 & 96.72 \\
\hline DRIVE (40) & 17.70 & 7.59 & 7.74 & 18.66 & 4.07 & 100 & 100 \\
\hline DIARETDB1 (89) & 11.95 & 4.73 & 5.66 & 8.10 & 3.14 & 100 & 100 \\
\hline CVocal (63) & 3.23 & 1.89 & 5.23 & 6.13 & 4.15 & 100 & 100 \\
\hline$D M E D$ (169) & 4.22 & 1.87 & 1.83 & 3.91 & 1.21 & 99.40 & 100 \\
\hline MESSIDOR (100) & 4.95 & 1.85 & 3.57 & 4.24 & 2.57 & 100 & 100 \\
\hline Combined (579) & 7.72 & 3.74 & 5.76 & 8.03 & 3.94 & 99.13 & 99.65 \\
\hline
\end{tabular}

Our integrated method achieves an overall sensitivity of $99.13 \%$ and $99.65 \%$ for OD detection and macula detection respectively in Combined, which is comparable with [7] and state-of-the-art [3]. We get $\xi_{d i s c}\left(D^{*}\right)$ and $\xi_{m a c}\left(M^{*}\right)$ values 
of $3.74 \%$ and $3.94 \%$, which is considerably lower than $7 \%$ reported in [9]. On STARE dataset alone, the overall sensitivity of OD detection is $95 \%$, comparable to reported results of [8], whereas in macula detection, our method achieves sensitivity $97.53 \%$, an improvement over the best published results of [6]. Based on our extensive evaluation and results of the intermediate stages of our integration method, we tabulate the error percentages observed among true-positives in each dataset of our collection, along with Combined (shown in Table. 1). Three important patterns are evident: 1 . In disc-centered images, $\xi_{\text {mac }}\left(M_{2}\right)<\xi_{\text {mac }}\left(M_{1}\right)$ whereas in central view images, $\xi_{\text {mac }}\left(M_{2}\right)>\xi_{\text {mac }}\left(M_{1}\right), 2 . D^{*}$ and $M^{*}$ show considerably lower $\xi$ values than the individual estimates, for both the OD and macula localization across all datasets, 3. The normalized error $\xi_{\text {disc }}\left(D^{*}\right)$ and $\xi_{m a c}\left(M^{*}\right)$ is less than $5 \%$ in Combined.

Our analysis shows the merit of our integrated method over the individual estimates. The strongest attribute in our method is the iterative refinement of blood vessels, which provides $M_{1}$, and good initialization for twin parabola (yielding $D_{2}, M_{2}$ ). Though the percentage error of these estimates appear high compared to $D^{*}, M^{*}$, they provide accurate bounds within which intensity and anatomy information are applied, to give both accurate and precise locations $\left(D^{*}, M^{*}\right)$, thus yielding high detection sensitivity. This pattern is clearly seen in DRIVE, a non-mydriatic dataset having choroidal vessels.

On pathological datasets such as DMED (macular edema), DIARETDB1, MESSIDOR (DR), our method shows low error for $M^{*}$ with nil false-positives, indicating its potential in disease grading applications. In disc-centered images, the estimate $M_{2}$ is more reliable than $M_{1}$, as evident from table.1, since twin parabolas would fit the retinal arcades better than ellipse. Among the true positives, the error values appear to be relatively high in STARE, since it has images with indistinct or partial OD compounded by absence of parabolic focal chord vessels (fig.6(3)), disproportionate distance between OD and macula due to varied angles of acquisition.
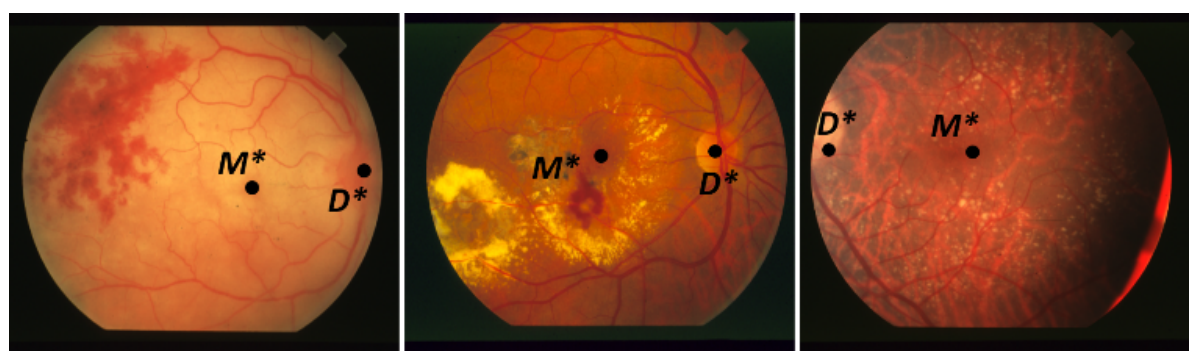

Fig. 6. (1) and (2) Accurate $D^{*}$ and $M^{*}$ in pathological images. (3) Accurate $M^{*}$ in spite of imprecise $D^{*}$

From the evaluation, we observe that our method performs satisfactorily with low $\xi_{d i s c}\left(D^{*}\right)$ and $\xi_{m a c}\left(M^{*}\right)$ even in case of imaging artifacts, presence of macular pathologies such as GA, bright lesion clusters and disc pathologies such as bleeding, hemorrhage on disc (fig. 6 (1 and 2)) and localizes macula accurately even with imprecise OD localization (fig. 6 (3)). 


\section{Conclusion}

The key contribution of our method is integration of multiple approaches such as intensity based segmentation, parametric modeling and anatomical cues using distance transform, by leveraging the strengths of individual approaches. The proposed algorithm for the detection of OD and macula is highly robust, giving $99 \%$ detection sensitivity for both OD and macula detection on highly diverse pathological dataset with overall normalized errors, $\xi_{\text {disc }}$ and $\xi_{m a c}$ of $3.74 \%$ and $3.94 \%$ respectively, thus showing reliable results, with immediate applicability in disease analysis. Our future work will target the challenges in analyzing other views of retina involving partial/absent OD and macula, leading to utility in multi-view analysis and registration, and OD segmentation for glaucoma analysis.

\section{References}

1. Zhu, X., Rangayyan, R.M., Ells, A.L.: Detection of the optic nerve head in fundus images of the retina using the hough transform for circles. J. Digital Imaging 23(3) (2010) 332-341

2. Abdel-Razik Youssif, A.H., Ghalwash, A.Z., Abdel-Rahman Ghoneim, A.: Optic disc detection from normalized digital fundus images by means of a vessels' direction matched filter. IEEE Trans. Medical Imaging 27(1) (2008) 11-18

3. Niemeijer, M., Abràmoff, M.D., van Ginneken, B.: Fast detection of the optic disc and fovea in color fundus photographs. MEDIA 13(6) (2009) 859-870

4. Hoover, A., Goldbaum, M.: Locating the optic nerve in a retinal image using the fuzzy convergence of the blood vessels. IEEE Trans. Medical Imaging 22(8) (2003) 951-958

5. Foracchia, M., Grisan, E., Ruggeri, A.: Detection of optic disc in retinal images by means of a geometrical model of vessel structure. IEEE Trans. Medical Imaging 23(10) (2004) 1189-1195

6. Lu, S., Lim, J.H.: Automatic macula detection from retinal images by a line operator. In: IEEE ICIP 2010. 4073-4076

7. Li, H., Chutatape, O.: Automated feature extraction in color retinal images by a model based approach. IEEE Trans. Biomed Engg, 51(2) (2004) 246-254

8. Tobin, K.W., Chaum, E., Govindasamy, V.P., Karnowski, T.P.: Detection of anatomic structures in human retinal imagery. IEEE Trans. Medical Imaging 26(12) (2007) 1729-1739

9. Lalonde, M., Beaulieu, M., Gagnon, L.: Fast and robust optic disc detection using pyramidal decomposition and hausdorff-based template matching. IEEE Trans. Medical Imaging 20(11) (2001) 1193-1200

10. Welfer, D., Scharcanski, J., Kitamura, C.M., Dal Pizzol, M.M., Ludwig, L.W., Marinho, D.R.: Segmentation of the optic disk in color eye fundus images using an adaptive morphological approach. CBM 40(2) (2010) 124-137

11. Tan, N., Wong, D., Liu, J., Ng, W., Zhang, Z., Lim, J., Tan, Z., Tang, Y., Li, H., Lu, S., et al.: Automatic detection of the macula in the retinal fundus image by detecting regions with low pixel intensity. In: ICBPE'09., IEEE (2009) 1-5

12. Köse, C., İkibaş, C.: Statistical techniques for detection of optic disc and macula and parameters measurement in retinal fundus images. Journal of Medical and Biological Engineering 31(6) (2011) 395-404 